\title{
Miíase bucal e doença de Alzheimer: relato de caso clínico
}

\author{
Oral myiasis and Alzheimer disease: report of a clinical case
}

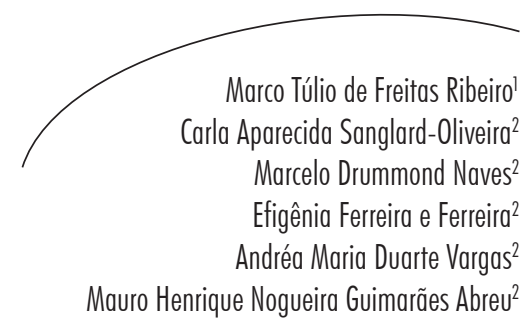

\section{Resumo}

Miíase é uma afecção causada pela presença de larvas de moscas em órgãos e/ou tecidos do homem ou de outros animais vertebrados, sendo mais frequentemente observada em países tropicais. A ocorrência de miíase na cavidade bucal é rara. Dentre os fatores predisponentes, destacam-se a senilidade, o comprometimento neurológico e a halitose. Assim, pacientes com doenças neuro-degenerativas, como a doença de Alzheimer (DA), são mais propensos a adquirirem essa alteração. Este trabalho relata um caso de miíase bucal, acometendo um paciente de 67 anos de idade, com DA em fase avançada, totalmente dependente para as atividades de vida diária (AVD), residente em uma instituição de longa permanência (ILP). O paciente apresentava falta de selamento labial e resistência aos cuidados de higiene bucal. O diagnóstico foi estabelecido clinicamente com base na observação de sangramento na região anterior do palato, descolamento da mucosa bucal e presença de larvas. Foi prescrito o uso de ivermectina e o paciente foi hospitalizado para debridamento do tecido necrótico e remoção das larvas. Após a alta, os cuidadores foram orientados quanto ao uso de máscara pelo paciente, que não se mostrou efetiva, pois o paciente passou a mordê-la, levando a seu deslocamento e reinfestação por larvas. Após o tratamento da reinfestação, adotou-se o uso de cortinado como rotina. Pacientes com DA constituem um grupo de risco para miíase bucal, sendo necessário orientar cuidadores e familiares em relação aos cuidados odontológicos para a prevenção desta patologia. O principal tratamento para a alteração baseia-se na remoção mecânica das larvas e instituição do uso oral da ivermectina.

\footnotetext{
Complexo de Reabilitação e Cuidado ao Idoso, Casa de Saúde Santa Izabel. Fundação Hospitalar do Estado de Minas Gerais. Betim, MG, Brasil.

2 Faculdade de Odontologia, Departamento de Odontologia Social e Preventiva. Universidade Federal de Minas Gerais. Belo Horizonte, MG, Brasil.
}

Palavras-chave: Miíase. Cavidade bucal. Idoso. Doença de Alzheimer. Prevenção.

Correspondência / Correspondence

Carla Aparecida Sanglard-Oliveira

Universidade Federal de Minas Gerais

Faculdade de Odontologia. Departamento de Odontologia Social e Preventiva.

Av. Antônio Carlos 6627, Pampulha

31270901 Belo Horizonte, MG, Brasil

E-mail: carlinhasanglard@yahoo.com.br 


\section{Abstract}

Myiasis is a disease caused by the presence of fly larvae in the organs and / or tissues of humans or other vertebrates, and is most often seen in tropical countries. The occurrence of myiasis in the oral cavity is rare. Among the predisposing factors stand out senility, neurological impairment and halitosis. Thus, patients with neurodegenerative diseases such as Alzheimer's disease (AD) are more likely to acquire this disorder. This paper reports a case of oral myiasis, involving a 67-year old patient with AD at an advanced stage, completely dependent for activities of daily living (ADL), residing in a long-term institution (ILP). The patient suffered from a lack of lip seal and resistance to oral hygiene care. The diagnosis was clinically made, based on the observation of bleeding in the anterior palate, detachment of the oral mucosa and presence of larvae. We prescribed the use of ivermectin and the patient was hospitalized for debridement of necrotic tissue and removal of the larvae. After hospital discharge, caregivers were oriented about the use of masks by the patient, which was not effective because the patient began to bite it, leading to its displacement and re-infestation by maggots. After treatment of re-infestation, it was adopted the routine use of drapery. AD patients are a risk group for oral myiasis, being necessary to direct caregivers and family members in relation to oral care to prevent this disease. The main treatment for the change is based on mechanical removal of larvae and instituting the use of oral ivermectin.
Key words: Myiasis. Mouth. Elderly. Alzheimer's disease. Prevention.

\section{INTRODUÇ̃̃̂O}

O termo "miíase" é derivado do grego myio (mosca) e ase (doença), e é usado para definir a invasão dos tecidos do corpo ou cavidade de animais vivos por ovos ou larvas de moscas da ordem díptera. O termo "berne" é também usado para esta doença. É frequente a ocorrência de miíase no meio rural em animais de criação, como bovinos, caprinos, camelídeos, suínos etc., e mesmo em animais domésticos, como cães e gatos, causando danos aos rebanhos e prejuízos econômicos. Pode também ocorrer em seres humanos, embora seja mais raro do que em animais. $^{1-4}$

As principais moscas causadoras dessa afecção em humanos são encontradas com frequência no Brasil, principalmente nas regiões de clima quente e úmido e na zona rural. ${ }^{4,5}$ As larvas de Cochliomyia hominivorax e Dermatobia são os agentes causais mais comuns das míases humanas na América. ${ }^{6}$

Pesquisa realizada com 24 pacientes com diagnóstico de míase, atendidos em três hospitais da rede pública na cidade do RecifePE, no período de novembro de 1999 a outubro de 2002, mostrou que em relação aos sítios anatômicos, as miíases ocorrem com maior frequência nos membros inferiores $(41,7 \%)$, com menor frequência na cabeça $(16,7 \%)$, cavidade bucal $(12,5 \%)$, ânus, ouvido e olho $(8,3 \%)$ e região dorsal $(4,2 \%)^{7}$

A ocorrência de miíase bucal é rara e há poucos relatos de casos clínicos na literatura descrevendo esta condição. Quando ocorrem, podem manifestar-se em bolsas periodontais, feridas resultantes de extrações dentárias e em diversas localizações dentro da cavidade bucal. ${ }^{8}$ As moscas depositam seus ovos diretamente sobre o tecido lesado. Depois de estes eclodirem, a larva obtém sua nutrição por meio de tecidos circunjacentes, e introduzem-se mais profundamente nos tecidos moles, podendo até produzir "túneis", separando a gengiva e o mucoperiósteo do osso. De acordo com a localização e o tecido envolvido, há diferentes nomenclaturas, como: miíase gengival, miíase dental, miíase periodontal e miŕase oral. ${ }^{9}$

Pacientes acometidos por miíase bucal em geral apresentam alguns fatores predisponentes, tais como: higiene bucal deficiente, falta de selamento dos lábios, resistência corporal diminuída, desnutrição, respiração bucal (principalmente durante o sono), etilismo, 
senilidade, comprometimento neurológico, hemiplegia e traumas na área facial. ${ }^{10} \mathrm{~A}$ halitose severa é citada como fator de risco, pois atrai as moscas para deposição das larvas. ${ }^{8}$ Esses fatores fazem com que pacientes com doença de Alzheimer (DA) sejam mais predispostos a desenvolver miíase. ${ }^{8,10}$

A DA é uma alteração neuro-degenerativa, progressiva e fatal, com perda da função intelectual, como memória, linguagem, localização espacial e temporal, habilidade para resolver problemas e de pensar de forma abstrata $\mathrm{e}$ com comportamento anormal. A doença aparece mais frequentemente após os 60 anos, englobando uma população com declínio na capacidade de apreender informações novas e de fazer tarefas rotineiras. Esses pacientes começam a perder a capacidade de se preocuparem com eles próprios e, em estágios avançados, perdem a mobilidade progressivamente, terminando com a morte. ${ }^{11,12}$ Ao longo do processo de demência, o paciente demonstra a perda progressiva da capacidade diária de higiene bucal (ADHB). Esta situação se deve a capacidade motora e funções cognitivas diminuídas, o que requer o cuidado especial desses indivíduos por um cuidador. ${ }^{13}$ Problemas bucais são mais prevalentes em subgrupos de idosos, como os institucionalizados, os funcionalmente dependentes e os demenciados. ${ }^{14}$ Indivíduos com necessidades especiais de cuidados de saúde possuem risco aumentado para doenças bucais, sendo que os pacientes DA constituem um exemplo desta situação. ${ }^{12}$

Devido ao processo evolutivo da doença, é essencial investigar as complicações que a DA pode produzir na saúde bucal, de modo que esses pacientes possam ser submetidos a procedimentos preventivos adequados para evitar o desenvolvimento e progressão de doenças bucais. Em relação à miíase bucal, compete ao cirurgião-dentista orientar familiares e cuidadores, diagnosticar e tratar prontamente esta afecção antes que ela chegue a um estado crítico, às vezes mortal, por hemorragias ou comprometimento do sistema nervoso central.

O objetivo deste trabalho é relatar um caso clínico sobre míase bucal em paciente idoso com doença de Alzheimer e discutir as medidas possíveis para prevenção e tratamento, de modo a orientar profissionais da saúde e cuidadores sobre a relevância dos cuidados com a saúde bucal nos casos da DA.

\section{RELATO DO CASO}

Paciente do sexo masculino, leucoderma, divorciado, 67 anos, portador de demência do tipo Alzheimer em fase avançada, totalmente dependente para as atividades de vida diária (AVD), afásico, residente em uma instituição de longa permanência (ILP). Solicitou-se ao cirurgião-dentista avaliação do paciente após cuidadoras observarem sangramento na região de palato duro e halitose. Ao exame clínico, observou-se que a gengiva e o mucoperiósteo apresentavam-se separados do tecido ósseo, com presença de sangramento e larvas (figura1). Foi diagnosticada míase bucal, prescrevendose ivermectina e cefalexina no momento do diagnóstico, sendo o paciente hospitalizado para curetagem e remoção das larvas (figuras 2 e 3).

Na ocasião, foram realizadas as exodontias dos elementos 22, 25 e 26. Após a remoção de tecido necrótico e larvas, observou-se cicatrização da lesão (figura 4). Os cuidadores foram orientados quanto ao uso de máscara no paciente, devido à ausência de selamento labial do mesmo. Tal conduta não se mostrou efetiva para a prevenção de reinfestação por larvas, pois o paciente adotou o hábito de morder a máscara, o que fez com que esta não permanecesse em posição.

Após sete meses, o paciente foi novamente diagnosticado com miíase bucal. Foi feita novamente a prescrição de ivermectina, irrigação com nitrofurazona (Furacinß) e remoção de larvas. A irrigação com nitrofurazona e a remoção de larvas mostraram-se insuficientes para a resolução do caso, devido a localização e quantidade de larvas presentes. O paciente foi então hospitalizado por uma semana, sendo medicado com amoxicilina e clavulanato de potássio, além de ivermectina (Ivomec ${ }^{\circledR}$ ). Durante esse período, foi feita diariamente a remoção das larvas. Após estas condutas, o 
paciente recebeu alta, sendo indicado o uso de levofloxacina $500 \mathrm{mg}$ por uma semana pelo médico infectologista que acompanhou o caso.

Uma semana após a alta hospitalar, o paciente compareceu à clínica da Faculdade de Odontologia da Universidade Federal de Minas Gerais para avaliação, quando se constatou extensa área de necrose. Cuidadoras foram orientadas a realizar higiene bucal com gluconato de clorexidina $0,12 \%$ diariamente, uso de máscara e cortinado. No

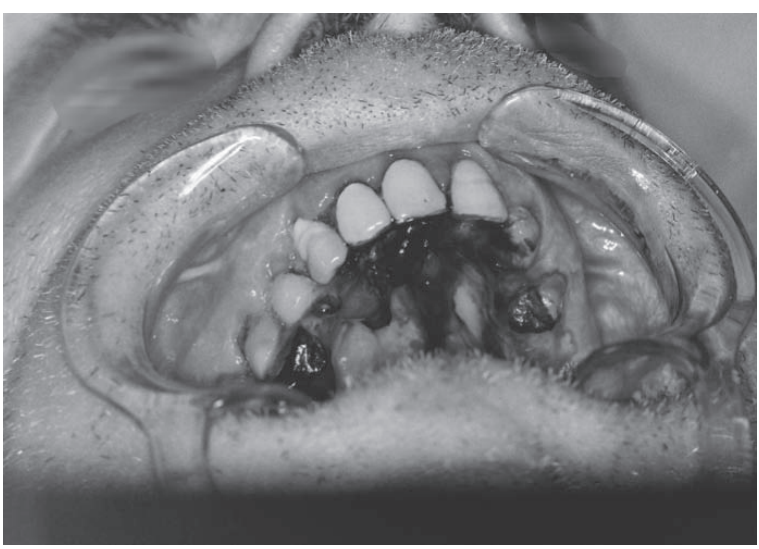

Figura 1 - Mucosa palatina descolada do periósteo com presença de sangramento e larvas.

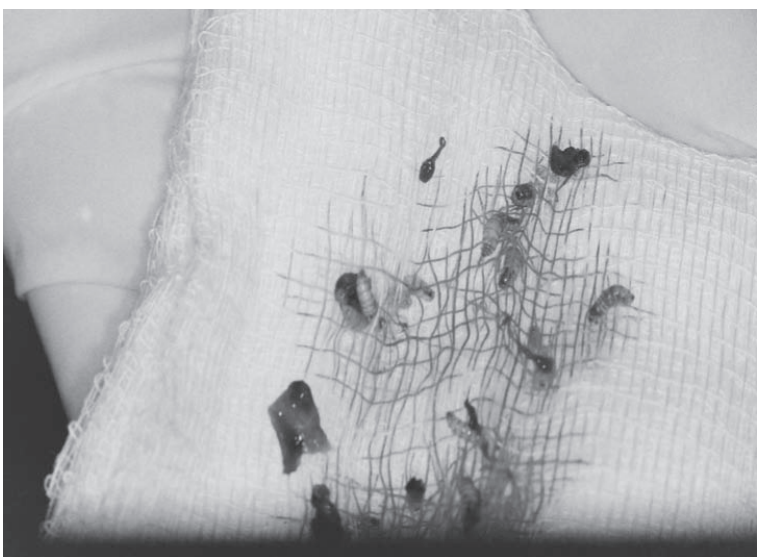

Figura 3 - Larvas removidas durante curetagem. mês seguinte, constatou-se cicatrização das áreas de necrose da região acometida (figura 4). $\mathrm{Na}$ ocasião, observou-se a presença de gengivite na região de incisivos inferiores, sendo os cuidadores novamente orientados quanto aos cuidados bucais.

O referido relato de caso recebeu autorização de familiares do paciente, sendo que o cuidador principal assinou o termo de consentimento livre e esclarecido, o qual permitia o uso da imagem e o relato do caso para publicação.

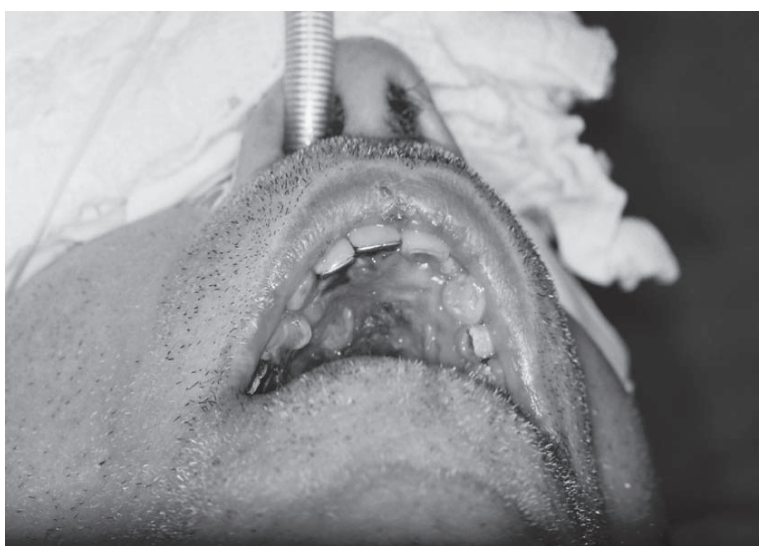

Figura 2 - Paciente entubado para debridamento de tecido necrótico e remoção de larvas.

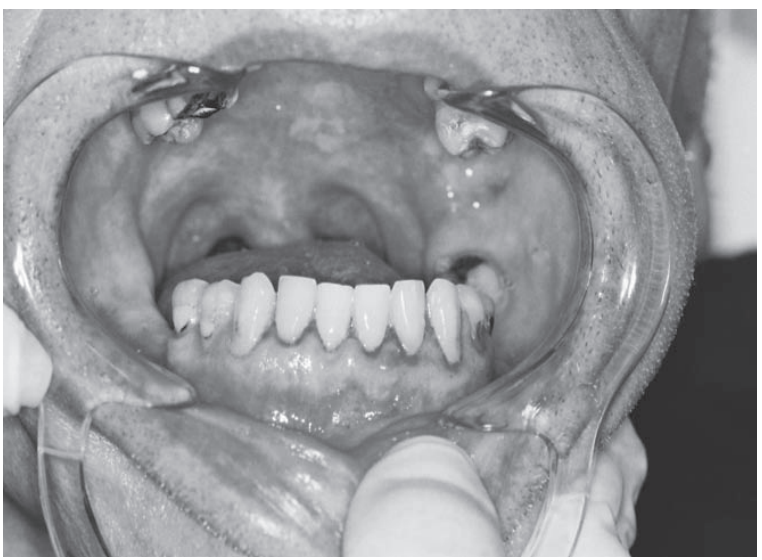

Figura 4 - Aspecto do palato após um mês. 


\section{DISCUSSÃO}

O Brasil encontra-se num processo de transição demográfica e epidemiológica, com aumento do número de idosos e da prevalência de doenças neuro-degenerativas como a DA. ${ }^{15}$ As dificuldades com as $\mathrm{ADHBs}$ devido a DA, associadas ao fato do Brasil ser uma região propícia ao desenvolvimento das principais espécies de moscas que causam miíase em humanos, favorecem o desenvolvimento desta patologia neste grupo de pacientes.

As condições de higiene da instituição são boas. É uma instituição classificada como boa ou muito boa pela Promotoria de Defesa da Pessoa Portadora de Deficiência e Idosos (PDPPDI), após avaliação quanto aos seguintes critérios: condições de moradia, limpeza e higiene do local, alimentação, equipe de saúde própria, atividades de lazer e recreativas, garantia aos idosos de seus direitos previdenciários, regulamentação legal, programa de manutenção de vínculos familiares, programa de capacitação e treinamento dos funcionários e cuidadores e atender especificamente pessoas com 60 anos ou mais. ${ }^{16}$

A ocorrência de miíase é mais comum em pessoas idosas, debilitadas, doentes e com deficiência mental. ${ }^{3,4}$ Assim, profissionais envolvidos no cuidado desses pacientes devem conhecer estratégias para prevenção, diagnóstico e tratamento de tal doença. A míase bucal, ainda que mais rara, é descrita em pacientes com higiene bucal deficiente, respiração bucal, falta de selamento dos lábios e halitose severa. Esses fatores atraem as moscas para deposição das larvas. ${ }^{17-19}$

No presente caso, o paciente, por ser portador da doença de Alzheimer em estágio avançado, é totalmente dependente das cuidadoras que trabal ham na ILP na qual ele vive. A dependência, associada às dificuldades com as ADHBs, a consequente halitose e falta de selamento labial do paciente, contribuíram para a infestação por larvas em sua cavidade bucal, ainda que esse viva em ambiente urbano com boas condições de higiene. Como o paciente é afásico, ele não pode externar os sintomas da infestação, sendo os sinais de anormalidade percebidos pelas cuidadoras somente quando a doença apresenta sinais clínicos. Assim, o mesmo demanda cuidados com as ADHB e uma rotina de avaliação da cavidade bucal como principal estratégia para a prevenção da infestação por larvas de moscas e outras patologias bucais. A clínica possuía um cirurgião-dentista de referência, que acompanhava os pacientes de acordo com a necessidade e por demanda da família. O paciente era acompanhado rotineiramente, recebendo visitas de um cirurgião-dentista semestralmente.

Além dos cuidados com as ADHBs, medidas de proteção individuais e coletivas são as principais formas para se prevenir a instalação de miíase. Entre elas, telar portas e janelas de hospitais ou dependências domésticas, que abriguem pacientes portadores de lesões ou de quaisquer condições predisponentes à instalação da miíase, evitar exposição de feridas abertas, ulcerações com tecido necrosado, eczemas infectados, assim como manter boa higiene individual e ambiental, são procedimentos de suma importância. ${ }^{18}$

$\mathrm{Na}$ instituição onde o paciente residia, não existiam telas nas janelas ou portas; entretanto, após a infestação da miíase na cavidade oral do mesmo, foram instalados cortinados no quarto do paciente, e estes foram mantidos mesmo após a resolução da infecção. A ausência de selamento labial e a colaboração do paciente devido à DA, associada à falta de informação das cuidadoras sobre as medidas preventivas para se evitar o aparecimento e recidiva da miíase, contribuíram para ainfestação no caso descrito. Cabe, entretanto, ressaltar a complexidade dos cuidados bucais preventivos nos pacientes com DA, pois ainda que o uso de máscaras tenha sido recomendado às cuidadoras para evitar a recidiva da míase, havia dificuldade da mesma em permanecer na face do paciente, pois este passou a mordê-la, provocando seu deslocamento. A incapacidade de compreensão e colaboração do paciente, tanto com as $\mathrm{ADHBs}$ quanto com estratégias para prevenção da reinfestação por larvas, constituiu um desafio para os profissionais envolvidos em 
seus cuidados, sendo necessária sua adequação à condição do paciente e do ambiente.

Quanto aos meios de tratamento, todos os estudos citados apontaram para a remoção mecânica das larvas como sendo o procedimento de escolha em pacientes acometidos pela míase bucal. Em casos muito severos, pode ser feita antibioticoterapia sistêmica. A ivermectina oral foi o medicamento antiparasitário de escolha usado para o tratamento de mí́ase bucal nos estudos de Ribeiro et al. ${ }^{20}$ e Shinohara et al., ${ }^{21}$ segundo os quais foram obtidos resultados considerados eficientes.

Fares et al., ${ }^{22}$ em relato de caso, tratando um paciente de dez anos de idade portador de deficiência mental e paraplégico, concluiu que a miíase bucal é predominante em população rural e em pacientes especiais, com debilidade mental, senilidade avançada, caquexia, dentre outros. Quanto ao tratamento, os bons resultados obtidos deveram-se mais à remoção mecânica das larvas do que à terapêutica medicamentosa empregada, quer seja local ou sistêmica.

No caso relatado, foi realizada remoção mecânica das larvas e instituição de um tratamento medicamentoso com ivermectina, o que condiz com o tratamento para miíase proposto pela literatura. O paciente, porém, foi novamente infestado por larvas e, devido à severidade e profundidade da lesão, outros medicamentos antibióticos como a amoxicilina associada a clavulanato de potássio e cefalexina foram prescritos ao paciente, para auxiliar no combate à infestação.

Recentemente, a irrigação com nitrofurazona tem sido apontad a como indicada para tratamento da miíase bucal, mas neste caso a irrigação não se mostrou eficaz, devido a profundidade da lesão e dificuldade no manuseio do paciente.

\section{CONCLUSÃO}

Pacientes com doença de Alzheimer constituem um grupo de risco para miíase bucal, e a prevenção dessa patologia constitui um desafio para cuidadores e familiares, especialmente no caso de pacientes com ausência de selamento labial.

A informação e orientação para familiares, cuidadores e para as instituições de longa permanência em relação aos cuidados bucais e ambientais e a avaliação rotineira da condição bucal desses pacientes constituem as principais estratégias preventivas para esse grupo. O principal tratamento paliativo para a alteração baseia-se na remoção mecânica das larvas e instituição do uso oral da ivermectina.

\section{REFERÊNCIAS}

1. James MT. The flies that cause myiasis in man. U.S. Depart. Agricul 1947; 631: 1-175.

2. Neves DP. Parasitologia Humana. 7 ed. Rio de Janeiro: Atheneu; 1988.

3. Desruelles F, et al. Myiasis caused by Dermatoba hominis after an organized tours in Amazonia. Presse Med 1999; 28(40): 2223-5.

4. Sherman RA. Wound myiasis in urban and suburban United States. Arch Intern Med 2000; 160(13): 2004-14.

5. Pizzoli LML, et al. Miíase: Uma experiência dramática em assistência de enfermagem. Rev Panam Infectol 2008; 10(3): 43-44.

6. Guimarães JH, Papavero N. Myiasis in man and animals in the Neotropical Region: bibliographic database. São Paulo: Editora Plêiade; 1999. 308 p.

7. Nascimento EMF et al. Míases humanas por Cochliomyia hominivorax (coquerel, 1858) (diptera, calliphoridae) em hospitais públicos na cidade do Recife, Pernambuco, Brasil. Entomol. Vect 2005; 12 (1): 37-51.

8. Novelli MR, Haddock A, Eveson JW. Orofacial myiasis. Br J Oral maxillofac Surg 1993; 31(1): 36-37.

9. Felices RR, Ogbureke KUE. Oral myiasis: report of case and review of management. J Oral Maxillofac Surg 2006; 54: 219-220 
10. Stephan A, Fuentefria NB. Miíase oral: parasita versus hospedeiro. Rev Ass Paul Cir Dent 1999; 53(1): 47-49.

11. Cummings JL. Alzheimer's disease. N Engl J Med 2004; 351: 56-67.

12. Friedlander AH, Norman DC, Mahler ME. Alzheimer's disease Psychopathology, medical management and dental implications. JADA 2006;137(9): 1240-51.

13. Goiato MC, et al. Odontogeriatria e a doença de Alzheimer. Pesq Bras Odontoped Clin Integr 2006; 6: 207-12.

14. Pearson A, Chalmers J. Oral hygiene care for adults with dementia in residential aged facilities. JBI Reports 2004; 2(3): 65-113.

15. Veras RP, Parahyba MICA. O anacronismo dos modelos assistenciais para os idosos na área da saúde: desafios para o setor privado. Cad. Saúde Pública, Rio de Janeiro 2007 out; 23(10): 2479-89.
16. Bozzo L, et al. Oral myiasis caused by sarcophagidae in an extraction wound. Oral Surg Oral Méd Pathol Oral Radiol Endod. 1992; 74(4): 733-5.

17. Pontes FSC, et al. Miíase gengival: relato de um caso clínico. JBC J Brás Clin Odontol Integr 2002; 6(32): 151-3.

18. Gomes de Araújo RJ, et at. Cochliomyia homnivorax em estágio avançado na cavidade oral. RGO, Porto Alegre 2009; 57(2): 229-233.

19. Ribeiro KC, et al. Tratamento da miíase humana cavitária com ivermectina oral. Rev Brás Otorrinolaringol 2001; 67(6): 755-61.

20. Shinohara EH, et al. Oral myiasis treated with ivermectin: case report. Braz Dent J 2004; 15(1): 79-81.

21. Fares NH, et al. Míase em paciente com 10 anos de idade: relato de caso clínico e revisão de literatura. Rev. de Clín. Pesq. Odontol. 2005; 1(4): 49-54.

22. MINAS GERAIS. Ministério Público - Promotoria de Defesa da Pessoa Portadora de Deficiência e do Idoso. 2000. 